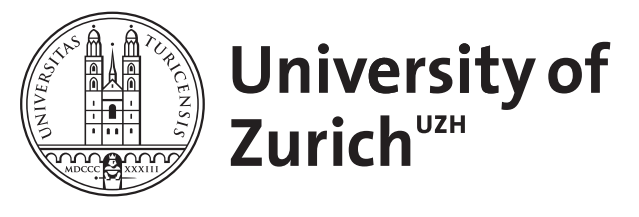

\title{
Gravitational lensing and rotation curve
}

\author{
Bräunlich, Gerhard ; Scharf, Günter
}

\begin{abstract}
Based on the geodesic equation in a static spherically symmetric metric we discuss the rotation curve and gravitational lensing. The rotation curve determines one function in the metric without assuming Einstein's equations. Then lensing is considered in the weak field approximation of general relativity. From the null geodesics we derive the lensing equation. The gravitational potential U(r) which determines the lensing is directly give by the rotation curve $U(r)=-v 2(r)$. This allows to test general relativity on the scale of galaxies where dark matter is relevant
\end{abstract}

DOI: https://doi.org/10.1007/s10714-010-1077-2

Posted at the Zurich Open Repository and Archive, University of Zurich

ZORA URL: https://doi.org/10.5167/uzh-155692

Journal Article

Published Version

Originally published at:

Bräunlich, Gerhard; Scharf, Günter (2011). Gravitational lensing and rotation curve. General Relativity and Gravitation, 43(1):143-154.

DOI: https://doi.org/10.1007/s10714-010-1077-2 


\title{
Gravitational lensing and rotation curve
}

\author{
Gerhard Bräunlich • Günter Scharf
}

Received: 15 March 2010 / Accepted: 12 August 2010 / Published online: 24 August 2010

(C) Springer Science+Business Media, LLC 2010

\begin{abstract}
Based on the geodesic equation in a static spherically symmetric metric we discuss the rotation curve and gravitational lensing. The rotation curve determines one function in the metric without assuming Einstein's equations. Then lensing is considered in the weak field approximation of general relativity. From the null geodesics we derive the lensing equation. The gravitational potential $U(r)$ which determines the lensing is directly give by the rotation curve $U(r)=-v^{2}(r)$. This allows to test general relativity on the scale of galaxies where dark matter is relevant.
\end{abstract}

Keywords Gravitational lensing · Geodesic flow

\section{Introduction}

As long as the dark matter problem is open there is a non-zero probability that general relativity might not hold on the scale of galaxies [1-5]. Therefore a direct test on this scale is highly desired. It is the purpose of this paper to show how such a test is possible, if kinematical and lensing data of the galaxy are available. The idea is the following: the rotation curve determines the time-time component of the metric without assuming Einstein's field equations, only the geodesic equation is used. Assuming now for the lensing the weak field approximation to general relativity where the metric is given in terms of the Newtonian gravitational potential $U(r)$ we find the extremely simple relation

$$
U(r)=-v^{2}(r)
$$

G. Bräunlich · G. Scharf $(\varangle)$

Institut für Theoretische Physik, Universität Zürich, Winterthurerstr. 190,

8057 Zürich, Switzerland

e-mail: scharf@physik.unizh.ch 
In contrast to the usual way of analyzing the data we get this result without assuming a matter model for the galaxy. This offers the possibility to test the basic physics in a situation where dark matter is important.

We treat lensing by means of the geodesic equation as well. By computing the null geodesics we derive the lensing equation. The most important part of the light path is the neighborhood of the deflector where the curvature is maximal. In standard lens theory the light path is approximated by two straight rays with a kink, but this approximation is particularly bad at the apex. Therefore, it is important to calculate the exact geodesics.

If the circular velocity $v(r)$ can be measured in the vicinity of the apex, then from the above relation (1.1) an expansion of $U(r)$ of the form

$$
U(r)=C_{0}+\frac{C_{1}}{r}+\frac{C_{2}}{r^{2}}
$$

can be obtained. That is all what is needed to calculate the lensing observables (see Sect. 3 below). Comparison with observations then tests general relativity in a modelindependent way (see Sect. 4).

\section{Geodesic flow and rotation curve}

We consider a static spherically symmetric metric which we write in the form

$$
d s^{2}=e^{v} c^{2} d t^{2}-e^{\lambda} d r^{2}-r^{2}\left(d \vartheta^{2}+\sin ^{2} \vartheta d \varphi^{2}\right)
$$

where $v$ and $\lambda$ are functions of $r$ only. We take the coordinates $x^{0}=c t, x^{1}=r$, $x^{2}=\vartheta, x^{3}=\varphi$ such that

$$
\begin{aligned}
& g_{00}=e^{v}, \quad g_{11}=-e^{\lambda} \\
& g_{22}=-r^{2}, \quad g_{33}=-r^{2} \sin ^{2} \vartheta
\end{aligned}
$$

and zero otherwise. The components with upper indices are the inverse of this. The determinant comes out to be

$$
g=\operatorname{det} g_{\mu \nu}=-e^{\nu+\lambda} r^{4} \sin ^{2} \vartheta
$$

Let us recall the Christoffel symbols for the metric (2.1) from the appendix of [4]

$$
\begin{aligned}
& \Gamma_{10}^{0}=\frac{1}{2} v^{\prime}, \quad \Gamma_{00}^{1}=\frac{1}{2} v^{\prime} e^{\nu-\lambda}, \quad \Gamma_{11}^{1}=\frac{1}{2} \lambda^{\prime}, \quad \Gamma_{22}^{1}=-r e^{-\lambda} \\
& \Gamma_{33}^{1}=-r e^{-\lambda} \sin ^{2} \vartheta, \quad \Gamma_{12}^{2}=\frac{1}{r}, \quad \Gamma_{33}^{2}=-\sin \vartheta \cos \vartheta, \quad \Gamma_{13}^{3}=\frac{1}{r}, \quad \Gamma_{23}^{3}=\cot \vartheta
\end{aligned}
$$


and zero otherwise, the prime denotes the derivative with respect to $r$ always. The geodesic equation is given by

$$
\frac{d^{2} x^{\alpha}}{d s^{2}}+\Gamma_{\beta \gamma}^{\alpha} \frac{d x^{\beta}}{d s} \frac{d x^{\gamma}}{d s}=0
$$

The origin of our reference frame is in the center of the galaxy. We consider geodesics in the plane $\theta=\pi / 2$, then we must solve the following three equations

$$
\begin{gathered}
\frac{d^{2} c t}{d s^{2}}+v^{\prime} \frac{d c t}{d s} \frac{d r}{d s}=0 \\
\frac{d^{2} r}{d s^{2}}+\frac{v^{\prime}}{2} e^{\nu-\lambda}\left(\frac{d c t}{d s}\right)^{2}+\frac{\lambda^{\prime}}{2}\left(\frac{d r}{d s}\right)^{2}-r e^{-\lambda}\left(\frac{d \varphi}{d s}\right)^{2}=0 \\
\frac{d^{2} \varphi}{d s^{2}}+\frac{2}{r} \frac{d r}{d s} \frac{d \varphi}{d s}=0
\end{gathered}
$$

Multiplying (2.6) by exp $v$ we find

$$
\frac{\partial}{d s}\left(e^{v} \frac{d c t}{d s}\right)=0
$$

so that

$$
\begin{array}{r}
e^{v} \frac{d c t}{d s}=\text { const. }=a \\
\frac{d c t}{d s}=a e^{-v} .
\end{array}
$$

Next multiplying (2.8) by $r^{2}$ we get

$$
r^{2} \frac{d \varphi}{d s}=\text { const. }=J
$$

where $J$ is essentially the conserved angular momentum, hence

$$
\frac{d \varphi}{d s}=\frac{J}{r^{2}}
$$

Finally, substituting (2.9) and (2.10) into (2.7) and multiplying by $2(\exp \lambda) \times d r / d s$ we obtain

$$
\frac{d}{d s}\left[e^{\lambda}\left(\frac{d r}{d s}\right)^{2}-a^{2} e^{-v}+\frac{J^{2}}{r^{2}}\right]=0
$$


Consequently, the square bracket is equal to another constant $=b$. Then the resulting differential equation can be written as

$$
\left(\frac{d r}{d s}\right)^{2}=a^{2} e^{-(\lambda+v)}+e^{-\lambda}\left(b-\frac{J^{2}}{r^{2}}\right) .
$$

To obtain the connection with the rotation curve which is an important astronomical observable, we remember the definition of the unitary 4-velocity

$$
u^{\alpha}=\frac{d x^{\alpha}}{d s} .
$$

The term unitary indicates that $u^{\alpha}$ has invariant length 1 :

$$
u^{2}=g_{\alpha \beta} \frac{d x^{\alpha}}{d s} \frac{d x^{\beta}}{d s}=\frac{(d s)^{2}}{(d s)^{2}}=1
$$

In our case $u^{\alpha}$ is equal to

$$
u^{\alpha}=\left(\frac{d c t}{d s}, \frac{d r}{d s}, 0, \frac{d \varphi}{d s}\right) .
$$

Using (2.9), (2.10) and (2.12) we easily see that

$$
u^{2}=-b=1
$$

which by (2.13) fixes the constant of integration $b=-1$.

Clearly the last constant of integration $a^{2}$ must be related to the geometry of the geodesics. To see this we consider the streamlines $r=r(\varphi)$. Dividing (2.12) by $J=r^{2} d \varphi / d s$ we obtain

$$
\left(\frac{1}{r^{2}} \frac{d r}{d \varphi}\right)^{2}=\frac{a^{2}}{J^{2}} e^{-(\lambda+v)}+e^{-\lambda}\left(\frac{b}{J^{2}}-\frac{1}{r^{2}}\right) .
$$

Introducing the variable

$$
w(\varphi)=\frac{1}{r(\varphi)},
$$

we write the equation in the form

$$
\left(\frac{d w}{d \varphi}\right)^{2}=\frac{a^{2}}{J^{2}} e^{-(\lambda+v)}+e^{-\lambda}\left(\frac{b}{J^{2}}-w^{2}\right) .
$$


To compare this equation with Newtonian dynamics we use the expansion of the metric for large $r$ :

$$
e^{-(\lambda+\nu)}=1+O\left(r^{-2}\right), \quad e^{-\lambda}=1-\frac{r_{s}}{r}+O\left(r^{-2}\right)
$$

Here

$$
r_{s}=\frac{2 G M}{c^{2}}
$$

is the Schwarzschild radius in case of a point mass. Then to order $1 / r$ we have

$$
\left(\frac{d w}{d \varphi}\right)^{2}+w^{2}=\frac{a^{2}+b}{J^{2}}-r_{S} w\left(\frac{b}{J^{2}}-w^{2}\right) .
$$

In Newtonian mechanics the bounded streamlines are ellipses

$$
\tilde{w}=\frac{1}{r}=\frac{1}{p}(1+e \cos \varphi),
$$

where $p$ and $e$ are parameter and eccentricity of the ellipse. $p$ is connected with the non-relativistic angular momentum $\tilde{J}$ by

$$
\frac{\tilde{J}^{2}}{p}=G M
$$

The Newtonian equation which corresponds to (2.20) now reads

$$
\left(\frac{d \tilde{w}}{d \varphi}\right)^{2}+\tilde{w}^{2}=\frac{e^{2}-1}{p^{2}}+\frac{2}{p} \tilde{w} .
$$

Comparing the coefficients in (2.20) and (2.23) we first find

$$
-r_{S} \frac{b}{J^{2}}=\frac{2}{p}
$$

By (2.22) and $\tilde{J}=c J$ this gives $b=-1$ in agreement with (2.15). Secondly, from

$$
\frac{a^{2}+b}{J^{2}}=\frac{e^{2}-1}{p^{2}}=-\frac{1}{p \tilde{a}},
$$

where $\tilde{a}$ is the big half-axis of the ellipse, we obtain by (2.22)

$$
a^{2}=1-\frac{G M}{c^{2} \tilde{a}}=1-\frac{r_{S}}{2 \tilde{a}} .
$$

This shows that $a^{2}$ is connected with the big half-axis of the Kepler ellipse. 
The 3-velocity $\vec{v}$ which is measured by astronomers is defined as

$$
\vec{v}=\left(\frac{d x^{1}}{d t}, \frac{d x^{2}}{d t}, \frac{d x^{3}}{d t}\right) .
$$

Using

$$
\frac{d s}{d t}=\frac{c}{a} e^{v}
$$

we can calculate

$$
\vec{v}^{2}=\vec{u}^{2}\left(\frac{d s}{d t}\right)^{2}
$$

where $\vec{u}^{2}$ is the spatial part in (2.13). Since our metric is diagonal it is simply given by

$$
-\vec{u}^{2}=\sum_{j=1}^{3} g_{j j} \frac{d x^{j}}{d s} \frac{d x^{j}}{d s}=1-a^{2} e^{-v}
$$

By (2.26) we now get the desired velocity squared

$$
\vec{v}^{2}=c^{2}\left(e^{v}-\frac{e^{2 v}}{a^{2}}\right) .
$$

As a check we determine the asymptotic behavior for large $r \gg r_{S}$. Assuming circular motion $(\tilde{a}=r)$ and using

$$
a^{2}=1-G M / c^{2} r
$$

we find

$$
\vec{v}^{2}=c^{2}\left(-v(r)-\frac{G M}{c^{2} r}+O\left(r^{-2}\right)\right) .
$$

Since $v=-r_{s} / r$ we arrive at

$$
\vec{v}^{2}=\frac{G M}{r}
$$

$M$ is the total mass (normal plus dark). This agrees with Newtonian dynamics (Kepler's third law). Summing up, the relation between observational quantities and 
theory is very direct. The rotation curve $v(r)$ gives the metric function $v(r)$ by solving the quadratic equation (2.29)

$$
e^{v}=\frac{a^{2}}{2}\left(1 \pm \sqrt{1-\frac{4}{a^{2}} \frac{v^{2}}{c^{2}}}\right)
$$

Using (2.30) and the Newtonian value (2.31) we have

$$
a^{2}=1-\frac{v^{2}}{c^{2}}
$$

Then for velocities $v \ll c$ we obtain from (2.32) the simple result

$$
e^{v}=1-2 \frac{v^{2}}{c^{2}}
$$

We see that we must take the plus sign in (2.32).

\section{Null geodesics and lensing}

In the case of null geodesics describing light rays the integration constant $b$ in the geodesic equation (2.16) must be 0

$$
\frac{1}{r^{4}}\left(\frac{d r}{d \varphi}\right)^{2}=e^{-\lambda}\left(\frac{a^{2}}{J^{2}} e^{-v}-\frac{1}{r^{2}}\right)
$$

In the lensing problem one uses the weak field approximation to general relativity

$$
e^{-v}=1-\frac{2 U(r)}{c^{2}}, \quad e^{-\lambda}=1+\frac{2 U(r)}{c^{2}} \approx e^{v},
$$

where $U(r)$ is the gravitational potential. The latter can be obtained from the rotation velocity according to (2.34)

$$
U(r)=-v^{2}
$$

Introducing the quantity

$$
d=\frac{J}{a}
$$

in (3.1), the following first order equation remains to be solved

$$
\left(\frac{d r}{d \varphi}\right)^{2}=\frac{r^{4}}{d^{2}}-\left(1+\frac{2 U}{c^{2}}\right) r^{2}
$$






Fig. 1 Null geodesic for lensing

Here we have neglected the quadratic term in $U$ in accordance with (3.2). The meaning of $d$ becomes clear when we consider the trivial solution for $U=0$ :

$$
r=\frac{d}{\sin \varphi}
$$

It describes a straight line with distance $d$ from the origin in polar coordinates (Fig. 1). After inversion the equation (3.5) can simply be solved by quadrature:

$$
\frac{d \varphi}{d r}=\frac{ \pm 1}{r \sqrt{\left.\frac{r^{2}}{d^{2}}\right)-1-\frac{2 U}{c^{2}}}}
$$

The sign herein depends on the branch of the geodesic to be calculated.

In case of a point-mass (Schwarzschild) lens we have

$$
U(r)=-\frac{G M}{r}
$$

and from (3.6) we get an elliptic integral for the polar angle $\varphi(r)$ :

$$
\varphi(r)-\varphi_{0}= \pm d \int_{r_{0}}^{r} \frac{d r}{\sqrt{r^{4}-r^{2} d^{2}+r r_{s} d^{2}}}
$$

where we have again used the Schwarzschild radius $r_{s}$ (2.19). To reduce this integral to Legendre's normal form we need the four zeros $a_{1}, a_{2}, a_{3}, a_{4}$ of the quartic under the square root. It is convenient to expand everything in powers of $\varepsilon$ :

$$
\varepsilon=\frac{r_{s}}{d}
$$

which is a small parameter. Then we have the following four real roots

$$
a_{1}=d\left(1-\frac{\varepsilon}{2}\right), \quad a_{2}=\varepsilon d, \quad a_{3}=0, \quad a_{4}=-d\left(1+\frac{\varepsilon}{2}\right)
$$


up to $O\left(\varepsilon^{2}\right)$. The integral (3.8) is an incomplete elliptic integral of the first kind $F(\Phi, k)$ where the parameter $k$ is given by

$$
k^{2}=\frac{\left(a_{2}-a_{3}\right)\left(a_{1}-a_{4}\right)}{\left(a_{1}-a_{3}\right)\left(a_{2}-a_{4}\right)}=2 \varepsilon+O\left(\varepsilon^{2}\right)
$$

(see [5], vol. II, p. 310).

As first application we compute the Einstein deflection angle. The origin of our coordinate system is at the mass $M$, polar axis goes from $M$ to the observer (see Fig. 1). We integrate (3.8) from the apex $r_{0}$ to infinity which gives us the deflection angle $2\left(\varphi_{\infty}-\pi\right)$. The apex is defined by the condition

$$
\frac{d r}{d \varphi}=0
$$

which gives

$$
r_{0}=a_{1}
$$

Then we obtain

$$
\varphi_{\infty}-\frac{\pi}{2}=\mu d F\left(\Phi_{\infty}, k\right)
$$

where the Jacobian $\mu$ is equal to

$$
\mu=\frac{2}{\sqrt{\left(a_{3}-a_{1}\right)\left(a_{4}-a_{2}\right)}}=\frac{2}{d}\left(1-\frac{\varepsilon}{2}\right)
$$

The argument $\Phi_{\infty}$ follows from

$$
\sin ^{2} \Phi_{\infty}=\frac{a_{4}-a_{2}}{a_{4}-a_{1}}=\frac{1}{2}\left(1+\frac{3}{2} \varepsilon\right)=\frac{1}{2}\left(1-\cos \left(2 \Phi_{\infty}\right)\right)
$$

(see [5], vol. II, p. 310). The elliptic integral can be expanded for small $k$ as follows

$$
F(\Phi, k)=\Phi+\frac{k^{2}}{4}\left(\Phi-\frac{1}{2} \sin 2 \Phi\right)+O\left(k^{4}\right)
$$

(see [5], vol. II, p. 313). This finally gives Einstein's result

$$
\varphi_{\infty}=\pi+\varepsilon
$$

Next we want to derive the lens equation. In this problem the observer is not at infinity but in a finite distance $D_{d}$ from the lens. The light source is at a distance $D_{d s}$ at the other side of the lens and an amount $\eta$ off the optical axis (see Fig. 1); we use the same notation as in [6]. We have now to compute the null geodesics from the source 
at distance $D_{d s}$ through the apex $r=r_{0}$ to the observer at distance $D_{d}$. Then the polar angle $\tilde{\beta}=\frac{\eta}{D_{d s}}$ of the source follows from

$$
\pi-\tilde{\beta}=\mu d\left[F\left(\Phi_{d s}, k\right)+F\left(\Phi_{d}, k\right)\right]
$$

Here the angle $\Phi_{d}$ is given by

$$
\begin{aligned}
\sin ^{2} \Phi_{d} & =\frac{a_{4}-a_{2}}{a_{4}-a_{1}} \frac{D_{d}-a_{1}}{D_{d}-a_{2}} \\
& =\frac{1}{2}\left(1-\cos \left(2 \Phi_{d}\right)\right)=\frac{1}{2}\left[1+\frac{3}{2} \varepsilon-\Theta+\Theta^{2} \varepsilon\right]
\end{aligned}
$$

where $\Theta=d / D_{d}$ is the angle under which the observer sees the source. Then from (3.17) we obtain

$$
F\left(\Phi_{d}, k\right)=\frac{\pi}{4}+\varepsilon\left(\frac{1}{2}+\frac{\pi}{8}\right)-\frac{\Theta}{2}-\frac{\varepsilon \Theta}{4} .
$$

$F\left(\Phi_{d s}, k\right)$ is given by the same formula with $\Theta$ substituted by $\alpha=d / D_{d s}$. Now we find from (3.19)

$$
-\tilde{\beta}=-\Theta-\alpha+2 \varepsilon+O\left(\varepsilon^{2}\right) .
$$

The lens equation is usually written in terms of the angles

$$
\beta=\tilde{\beta} \frac{D_{d s}}{D_{s}}, \quad \Theta=\frac{r_{s}}{D_{d} \varepsilon}, \quad \alpha=\Theta \frac{D_{d}}{D_{d s}} .
$$

Then (3.22) gives the following lens equation

$$
\beta=\Theta-2 \frac{D_{d s}}{D_{s}} \frac{r_{s}}{D_{d} \Theta}
$$

for the point-mass lens which is the standard result ([7], p. 27).

Now we turn to the formulation of the lens equation in an arbitrary spherically symmetric metric. From (3.6) we have the following integral for the polar angle of the null geodesics:

$$
\varphi(r)-\frac{\pi}{2}= \pm d \int_{r_{0}}^{r} \frac{d r}{r \sqrt{r^{2}-(1+u) d^{2}}},
$$

where we have introduced the dimensionless gravitational potential

$$
u(r)=\frac{2}{c^{2}} U(r)
$$


Here $r_{0}$ is the apex and it is important to note that only the potential values for $r \geq r_{0}$ contribute. For $u=0$ the trivial lens equation $\beta=\Theta$ comes out, this follows from (3.24) for $r_{s}=0$. For $u \neq 0$ but $|u| \ll 1$ the modification of the result comes from the neighborhood of the apex. It is therefore good enough to expand the potential $u(r)$ in the vicinity of $r=r_{0}$. For this purpose we use the beginning of the multipole expansion

$$
u(r)=c_{0}+\frac{c_{1}}{r}+\frac{c_{2}}{r^{2}}
$$

The constant term is necessary in view of the flat rotation curves; note that the potential has an absolute normalization in (3.2). It is unimportant that (3.27) breaks down for small $r$ because we need $r \geq r_{0}$ only.

With the three terms in (3.27) we get an elliptic integral of the first kind again:

$$
\varphi(r)-\frac{\pi}{2}= \pm d \int_{r_{0}}^{r} \frac{d r}{\sqrt{G(r)}}= \pm \frac{\mu d}{\sqrt{1-c_{0}^{2}}} F(\Phi, k)
$$

Here the quartic is given by

$$
G(r)=r^{4}-\left(d^{2}+c_{0} d^{2}\right) r^{2}-c_{1} d^{2} r-c_{2} d^{2},
$$

the Jacobian $\mu$ and the parameter $k$ are the same as before (3.13), (3.15). The four zeros of $G(r)$ are obtained by solving the two quadratic equations

$$
1+u(r)=0, \quad r^{2}(1-u(r))-d^{2}=0 .
$$

This leads to

$$
\begin{aligned}
& a_{1}=\frac{c_{1}}{2\left(1-c_{0}\right)}+\sqrt{\frac{d^{2}+c_{2}}{1-c_{0}}+\frac{c_{1}^{2}}{4\left(1-c_{0}\right)^{2}}} \\
& a_{2}=-\frac{c_{1}}{2\left(1+c_{0}\right)}+\sqrt{\frac{c_{1}^{2}}{4\left(1+c_{0}\right)^{2}}-\frac{c_{2}}{1+c_{0}}} \\
& a_{3}=-\frac{c_{1}}{2\left(1+c_{0}\right)}-\sqrt{\frac{c_{1}^{2}}{4\left(1+c_{0}\right)^{2}}-\frac{c_{2}}{1+c_{0}}} \\
& a_{4}=\frac{c_{1}}{2\left(1-c_{0}\right)}-\sqrt{\frac{d^{2}+c_{2}}{1-c_{0}}+\frac{c_{1}^{2}}{4\left(1-c_{0}\right)^{2}}} .
\end{aligned}
$$

Then the lens equation is contained in the analogous equation to (3.19)

$$
\pi-\tilde{\beta}=\frac{\mu d}{\sqrt{1-c_{0}^{2}}}\left[F\left(\Phi_{d s}, k\right)+F\left(\Phi_{d}, k\right)\right] .
$$


The angles $\Phi_{d}, \Phi_{d s}$ are given by the same formula (3.20) as before. The appropriate expansion of the lens equation (3.31) depends on the particular values $c_{0}, c_{1}, c_{2}$ in (3.27).

\section{A test of general relativity}

Such a test is possible if for a spherical (E0) galaxy rotation curve and lensing data are available. From the rotation curve $v(r)$ we get the gravitational potential $U(r)$ according to (3.3). In practice only three velocity values are needed in order to fit $U(r)$ to the multipole expansion (3.27). The quality of the fit will be good if some velocity value $v(r)$ is measured near the apex $r_{0}$ of the deflected light ray. Of course one must replace the euclidean distances by angular diameter distances as usual. Then knowing the three parameters $c_{0}, c_{1}, c_{2}$ the lens equation (3.31) can be solved using the values (3.30) in the elliptic integrals. The calculated lensing data can then be compared with the observed ones. A discrepancy would be either due to the lack of spherical symmetry or to a failure of the weak field metric (3.2) which is a consequence of Einstein's equations. The second possibility is interesting in connection with the dark matter problem [5].

Galaxies with joint lensing and dynamical data can be found in the Sloan Lens ACS Survey (SLACS) and its follow-up project [9]. Unfortunately, until today only one system SDSSJ 2321-097 has been analyzed in detail. This is an early-type elliptic galaxy which cannot be approximated by a spherically symmetric metric. So we must extend our model-independent analysis to the elliptic case or hope that the astronomers come up with a E0 lens galaxy.

\section{References}

1. Milgrom, M.: MNRAS 326, 126 (2001)

2. Bekenstein, J.D.: Contem. Phys. 47(6), 387 (2006)

3. Mannheim, P.D.: ApJ 391 (1992) 429, astro-ph/0505266v2 (2005)

4. Horava, P.: Phys. Rev. D 79, 084008 (2009)

5. Scharf, G.: From massive gravity to modified general relativity, Gen. Relativ. Gravit. (2009) doi:10.1007/s10714-009-0864-0 and G. Scharf, Quantum Gauge Theories—spin one and two, Google Books, free access (2010)

6. Erdelyi, A., et al.: Higher transcendental functions. McGraw-Hill Book Co. Inc., New York (1953)

7. Schneider, P., Kochanek, C., Wambsganss, J.: Gravitational lensing: strong, weak and micro. Springer, Berlin (2006)

8. Schneider, P., Ehlers, J., Falco, E.E.: Gravitational lenses. Springer, Berlin (1992)

9. Czoske, O., Barnabè, M., Koopmans, L.V.E., Treu, T., Bolton, A.S.: MNRAS 384, 987 (2008) 